\title{
Mikroinstabilitäten vom Spiegeltyp in inhomogenen Plasmen
}

\author{
Von D. Pfirsch \\ Aus dem Max-Planck-Institut für Physik und Astrophysik, München \\ (Z. Naturforschg. 17 a, 361-870 [1962]; eingegangen am 9. August 1962)
}

\begin{abstract}
The canonical momentum in the direction of the electrical current or an other ordered motion is a constant of motion in the case of mirror type perturbations of plasma configurations in which the current and the magnetic field are perpendicular to each other and in which all the macroscopic quantities depend only on one coordinate in a rectangular system of coordinates, of which the metrical tensor depends also on that coordinate only. This fact enables one, to discuss rather simply the onset of instabilities of that kind of perturbations within the frame of a microscopic collisionless theory. Several examples like plane and cylindrical $z$-and $\theta$-pinches and plane periodic configurations without space charges or without currents are treated in this way. A special result is, that at least a very wide class of the nonlinear electrostatic waves, found by Bernstein, Green and KrUSKal $^{1}$ are unstable.
\end{abstract}

Die vorliegende Arbeit befaßt sich mit dem Problem des Einsetzens von gewissen Mikroinstabilitäten in inhomogenen 2-komponentigen Plasmen. Die makroskopischen Gleichgewichtsgrößen, wie Dichte, Magnetfeld, elektrisches Feld und auch der metrische Tensor, sollen nur von einer Koordinate in einem orthogonalen Koordinatensystem abhängen, in dem der Strom oder eine andere geordnete Bewegung nur eine Komponente in Richtung einer 2. Koordinate besitzen soll. Beispiele dafür sind ebene oder zylindrische $\Theta$-Pinche und nichtstabilisierte $z$-Pinche. Die diskutierten Störungen sollen in Richtung der geordneten Bewegung im Plasma konstant sein. Nach Furth, durch dessen Salzburger Vortrag $^{2}$ die hier durchgeführten allgemeineren Überlegungen angeregt wurden, kann man derartige Störungen als solche vom Spiegeltyp bezeichnen, die von Störungen entlang der Stromlinien, die man mit Rosenbluth $^{3}$ als Störungen vom Landau-Typ bezeichnen kann, spezifisch verschieden sind. Die Störungen vom Spiegeltyp haben im Gegensatz zu denen vom LaNDAU-Typ die Eigenschaft, den kanonischen Impuls in Richtung der geordneten Bewegung zu erhalten, wodurch es möglich ist, in einfacher Weise das Einsetzen von Instabilitäten dieses Typs zu diskutieren, und zwar in Form der Untersuchung der möglichen Existenz stationärer Nachbarlösungen eines Gleichgewichts, die den Übergang zu instabilen Lösungen darstellen. In diesem Sinn wird also ein hinreichendes Kriterium für Instabilität angegeben.

1 J. B. Bernstein, J. M. Green u. M. D. Kruskal, Phys. Rev. 108, 546 [1957].

2 H. P. Furth, Conf. on Plasma Physics and Controlled Nuclear Fusion, Salzburg 1961, CN-10, 174. Siehe auch die
Im 1. Abschnitt wird die Methode allgemein entwickelt, im 2. Abschnitt werden das thermodynamische Gleichgewicht sowie $z$ - und $\Theta$-Pinche als Beispiele behandelt, ausführlicher werden noch ebene Anordnungen ohne Raumladungen oder ohne Ströme diskutiert.

\section{Darstellung der Methode}

Die Theorie wird in zwei Schritten entwickelt. Zunächst werden stationäre Nachbarlösungen eines Gleichgewichts bestimmt; sodann wird untersucht, ob diese Lösungen einen Übergang zu instabilen Lösungen darstellen.

\subsection{Die stationären Nachbarlösungen eines Gleichgewichts}

In einem rechtwinkligen Koordinatensystem $q^{1}$, $q^{2}, q^{3}$, dessen metrischer Tensor nur von $q^{1}$ abhängt, werde ein im Gleichgewicht befindliches 2-Komponenten-Plasma der Teilchensorten a und $\mathrm{b}$ durch die beiden Hamilton-Funktionen

$$
H_{0}^{(v)}=H_{0}^{(v)}\left(q^{1}, p_{01}^{(v)}, p_{02}^{(v)}, p_{03}^{(\nu)}\right), \quad v=\mathrm{a}, \mathrm{b}
$$

als Funktion von $q^{1}$ und der Geschwindigkeiten $\dot{q}_{i}$ beschrieben. $p_{0 i}{ }^{(v)}$ sind die kanonischen Impulse jeder Teilchensorte im ungestörten Plasma. Ein Magnetfeld sei nur in 2-Richtung vorhanden, ein elektrisches Feld in 1-Richtung. Dann lassen sich Lösun-

neue Arbeit von H. P. Furth, Report UCRL 6907, Livermore, California 1962.

3 M. N. Rosenbluth, N. Rostoker u. N. A. Krall, Conf. on Plasma Physics and Controlled Nuclear Fusion, Salzburg 1961, CN-10, 174. 
gen der stationären VLasov-Gleichung in der Form

$$
f_{0}^{(v)}\left(H_{0}^{(v)}, p_{03}^{(v)}\right)
$$

schreiben; sie ergeben einen Strom oder eine andere geordnete Bewegung oder Anisotropie in 3-Richtung. Im folgenden soll diese Klasse von Gleichgewichtslösungen untersucht werden.

Wird eine Störung des elektrischen Potentials und des Vektorpotentials in der Form

$$
\Phi_{1}\left(q^{1}, q^{2}\right), \quad A_{13}\left(q^{1}, q^{2}\right)
$$

eingeführt, dann sind

$$
\begin{aligned}
H^{(v)} & =H_{0}^{(v)}\left(q^{1}, p_{0 i}^{(v)}\right)+e^{(v)} \Phi_{1}, \\
p_{3}^{(v)} & =p_{03}^{(v)}+\left(e^{(v)} / c\right) A_{13}
\end{aligned}
$$

neue Konstanten der Bewegung, so daß ein zu (2) benachbartes Gleichgewicht durch Verteilungsfunktionen

$$
f_{0}^{(v)}\left(H^{(v)}, p_{3}^{(v)}\right)
$$

beschrieben wird. Daraus folgt, daß

$$
f_{1}^{(v)}=\left(\Phi_{1} \frac{\partial}{\partial \Phi_{0}}+A_{13} \frac{\partial}{\partial A_{03}}\right) f_{0}^{(v)}\left(H_{0}^{(v)}, p_{03}^{(v)}\right)
$$

Lösungen der hinsichtlich der Störungen linearisierten VLasov-Gleichungen sind; sie stellen noch nicht die allgemeinsten Lösungen dieser Gleichungen dar, doch sind sie die für das Stabilitätsproblem interessanten, die allgemeinsten Lösungen unterscheiden sich von den Lösungen (6) nur durch Lösungen des homogenen Teils der linearisierten VLasov-Gleichungen, also durch Ausdrücke, die $\Phi_{1}$ und $A_{13}$ nicht enthalten. $\Phi_{0}$ und $A_{03}$ sind Potential und Vektorpotential der ungestörten Felder.

Die Funktionen (6) haben die Eigenschaft, daß sie durch einen nicht von der Teilchensorte und den Geschwindigkeiten abhängigen linearen Operator aus den ungestörten Lösungen der VLasov-Gleichung hervorgehen, so daß für die Änderungen von Ladungs- und Stromdichte gilt

$$
\begin{aligned}
& \varrho_{1}=\left(\Phi_{1} \frac{\partial}{\partial \Phi_{0}}+A_{13} \frac{\partial}{\partial A_{03}}\right) \varrho_{0}\left(\Phi_{0}, A_{03}\right) ; \\
& j_{13}=\left(\Phi_{1} \frac{\partial}{\partial \Phi_{0}}+A_{13} \frac{\partial}{\partial A_{03}}\right) j_{03}\left(\Phi_{0}, A_{03}\right),
\end{aligned}
$$

$\varrho_{0}$ und $j_{03}$ charakterisieren das makroskopische System. Damit beide Größen konsistent mit der Vlasov-Gleichung sind, müssen sie z. B. bei einem

${ }^{4}$ H. VöLK, Selbstkonsistente stationäre Lösungen der VLAsovGleichung für ein Zweikomponentenplasma, Diplomarbeit aus dem Institut für Theoretische Physik der Universität München 1961, unveröffentlicht. ebenen Problem aus einer erzeugenden Funktion $W\left(\Phi_{0}, A_{03}\right)$ folgen (siehe z. B. $\left.{ }^{4}\right)$ :

$$
\varrho_{0}=-\frac{\partial W\left(\Phi_{0}, A_{03}\right)}{\partial \Phi_{0}}, \quad j_{03}=\frac{\partial W\left(\Phi_{0}, A_{03}\right)}{\partial A_{03}} ;
$$

außerdem müssen natürlich $\Phi_{0}$ und $A_{03}$ den durch $Q_{0}$ und $j_{03}$ bestimmten Maxwellschen Gleichungen genügen. Mit (7) und den Maxwellschen Gleichungen erhält man schließlich als Gleichungen für $\Phi_{1}$ und $A_{13}$

$\operatorname{div} \operatorname{grad} \Phi_{1}=-4 \pi \varrho_{1}, \quad\left(\operatorname{rot} \operatorname{rot} \boldsymbol{A}_{1}\right)_{3}=\frac{4 \pi}{c} j_{13}$.

Kovariant geschrieben sehen diese Gleichungen folgendermaßen aus:

$$
\begin{aligned}
& \frac{\partial}{\partial q^{1}}\left(g^{11} V g\right) \frac{\partial \Phi_{1}}{\partial q^{1}}+g^{11} V g \frac{\partial^{2} \Phi_{1}}{\left(\partial q^{1}\right)^{2}} \\
& +g^{22} \sqrt{g} \frac{\partial^{2} \Phi_{1}}{\left(\partial q^{2}\right)^{2}}=-4 \pi \varrho_{1}, \\
& \frac{\partial}{\partial q^{1}}\left(\sqrt{g} g^{33} g^{11}\right) \frac{\partial A_{13}}{\partial q^{1}}+\sqrt{g} g^{33} g^{11} \frac{\partial^{2} A_{13}}{\left(\partial q^{1}\right)^{2}} \\
& +\sqrt{g} g^{33} g^{22} \frac{\partial^{2} A_{13}}{\left(\partial q^{2}\right)^{2}}=-\frac{4 \pi}{c} g^{33} j_{13}, \\
& g=\left\|g_{i k}\right\|=g_{11} g_{22} g_{33} .
\end{aligned}
$$

In 2-Richtung ist ein Ansatz möglich

$$
\Phi_{1}, A_{13} \sim \exp \left\{i k q^{2}\right\} .
$$

Die Gln. (9) bzw. $\left(9^{\prime}\right)$ stellen dann ein Eigenwertproblem dar mit $k$ als Eigenwert. Reelle Eigenwerte $k$ gehören zu den möglichen stationären Nachbarlösungen.

\subsection{Nichtstationäre Nachbarlösungen zu stationären Störungen}

Zu einem durch (10) definierten Wellenvektor $k$, der von einem diskreten Eigenwert für eine stationäre Störung um ein infinitesimales $\delta k$ abweicht, kann es nur nichtstationäre Lösungen der linearisierten Gleichungen geben. Im folgenden wird das zeitliche Verhalten von Lösungen für infinitesimale $\delta k$ untersucht und damit die Frage, ob die unter 1.1 diskutierten Störungen instabile Nachbarlösungen besitzen.

Die zeitabhängige Vtasov-Gleichung kann in der Form

$$
\frac{\partial f}{\partial t}+[H, f]=0
$$

geschrieben werden. Der Klammerausdruck bedeutet die Poisson-Klammer, $H$ ist die gestörte HamiltonFunktion; der Index für die Teilchensorten ist un- 
terdrückt. Für die Lösung wird angesetzt

$$
f=f_{0}\left(H, p_{3}\right)+\varphi ;
$$

$f_{0}$ ist dieselbe Funktion wie in Gl. (2) ; es ist aber hier bequemer, die Impulse und nicht die Geschwindigkeiten als Variable zu verwenden. Einsetzen von (12) in (11) führt dann in linearer Näherung auf

$$
\begin{gathered}
\frac{\partial \varphi}{\partial t}+\frac{\partial H_{1}}{\partial t} \frac{\partial f_{0}}{\partial H}+\left[H_{0}, \varphi\right]=0, \\
H_{1}=e \Phi_{1}-(e / c) \boldsymbol{A}_{1} \boldsymbol{v} ;
\end{gathered}
$$

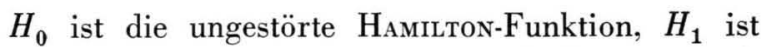
deren Störung durch Potential und Vektorpotential. Für $\left[H_{0}, \varphi\right]$ kann auch geschrieben werden

$$
\left[H_{0}, \varphi\right]=\mathcal{H}_{0} \varphi \text {, }
$$

wobei dann $\mathcal{H}_{0}$ ein linearer antihermitescher Operator ist. Seine Eigenwerte sind daher rein imaginär. Außerdem bilden sie ein Kontinuum. Da nämlich $H_{0}$ die Koordinate $q^{2}$ nicht enthält, ist $\mathcal{H}_{0}$ nach $q^{2}, p_{2}$ separierbar, so daß die Eigenfunktionen bezüglich $q^{2}$ und $p_{2}$ proportional zu

$$
\exp \left\{i k q^{2}\right\} \delta\left(p_{2}-P_{2}\right)
$$

sind, woraus additive Bestandteile der Eigenwerte der Größe

$$
(i / m) P_{2} k
$$

folgen. Dem Ansatz (10) entspricht ein fester Wert $k$, so daß für $k \neq 0$ das Kontinuum durch $P_{2}$ beschrieben wird.

Mit dem Zeitansatz

$$
\varphi, \Phi_{1}, \boldsymbol{A}_{1} \sim e^{i \omega t}
$$

folgt aus (13) und (14)

$$
\varphi=-\underset{i \omega+\mathcal{H}_{0}}{i \omega} H_{1} \frac{\partial f_{0}}{\partial H} .
$$

Ladungs- und Stromdichten ergeben sich hieraus durch entsprechende Integrationen über den Geschwindigkeitsraum. Da $\mathcal{H}_{0}$ nur rein imaginäre Eigenwerte besitzt, die für $k \neq 0$ durch (16) beschriebene kontinuierliche Bestandteile enthalten, liegen für reelle $\omega$ in (18) Pole bezüglich $p_{2}$ oder $v_{2}$ vor. Im Sinne eines Anfangswertproblems sind die Integrationen zur Berechnung der Ladungs- und Stromdichten zunächst so durchzuführen, daß $\omega$ einen negativen Imaginärteil besitzt; von den so entstehenden Ausdrücken hat man dann für beliebige $\omega$ die analytischen Fortsetzungen zu nehmen. Dadurch ergeben sich beim Einsetzen der Ladungs- und Stromdichten in die MaxweLL-Gleichungen keine reellen Eigenwertgleichungen für $\omega$, außer wenn an den Polstellen von (18) das Residuum verschwindet. Es fragt sich dann, ob und wann diese Gleichungen instabile Lösungen besitzen können.

Im thermodynamischen Gleichgewicht z. B. gibt es für $k \neq 0$ sowohl für die longitudinalen wie für die transversalen Wellen nur gedämpfte Wellen. Wie im 1. Beispiel gezeigt wird, existieren in diesem Fall aber keine stationären Lösungen. Im Falle, daß es stationäre Störungen gibt, kann man Nachbarlösungen zu diesen im Rahmen einer Störungsrechnung diskutieren. Die in den linearisierten Gleichungen auftretenden Glieder proportional zu $\delta k$ oder $\omega$ oder Potenzen von beiden werden als Störungen behandelt, in denen die ungestörten, also vom stationären Nachbarproblem her bekannten Funktionen und $k$-Werte einzusetzen sind. Damit werden sie $\mathrm{zu}$ inhomogenen Termen in den MAxwelL-Gleichungen, deren homogener Teil identisch mit dem stationären Problem ist. Die Bedingung, $\mathrm{d} \Omega($ die inhomogenen Glieder zusammengenommen orthogonal zu den Lösungen der adjungierten homogenen Gleichung sind, ergibt die Dispersionsbeziehung in der niedrigsten Ordnung in $\delta k$. Wenn der Eigenwert $k$ für die stationäre Lösung verschieden von Null ist, ist sie wegen (18) im allgemeinen von der Form

$$
k \delta k \sim \omega ;
$$

der Proportionalitätsfaktor muß nach dem Vorangegangenen im allgemeinen komplex sein, wobei wegen der Invarianz gegenüber Zeitumkehr noch die schärfere Aussage gilt, daß er rein imaginär sein muß; denn der Imaginärteil des Proportionalitätsfaktors rührt, wie oben beschrieben, von den Polen im Ausdruck für $\varphi$ her. Die zeitumgekehrte Lösung, die durch $-\omega$ beschrieben werden muß, wird durch umgekehrtes Umfahren dieser Polstellen bei der $v_{2}$-Integration erhalten, wodurch aber nur der Imaginärteil und nicht auch der Realteil des Proportionalitätsfaktors sein Vorzeichen ändert, so daß der Realteil verschwinden muß. Es gibt also immer ein Vorzeichen von $\delta k$ bezüglich $k$, dem eine instabile Lösung entspricht.

Es könnte sein, daß das zu $\omega$ proportionale Glied verschwindet, so daß in (19) höhere Potenzen von $\omega$ stehen, dann gibt es natürlich auch immer instabile Lösungen. Das zu $k \mathrm{~d} k$ proportionale Glied kann dagegen nie verschwinden, wenn das homogene Problem rein hermitesch oder antihermitesch ist, da 
es dann aus dem Integral über das Absolutquadrat der stationären Störungslösung resultiert, und allgemeiner dann nicht, wenn die Lösungen des adjungierten homogenen Problems nicht sämtlich orthogonal zu den Lösungen des homogenen Problems sind. Die Orthogonalität ist dabei gemäß Gl. $\left(9^{\prime}\right)$ mit einer vom metrischen Tensor abhängigen $\mathrm{Ge}$ wichtsfunktion definiert, die für ein kartesisches Koordinatensystem 1 ist.

Ist $k=0$ der betrachtete Eigenwert des stationären Problems, dann hat in (19) statt $k \delta k$ nun $(\delta k)^{2}$ zu stehen. In diesem Fall ist es daher nicht in der oben geschilderten einfachen Weise möglich, auf die Existenz instabiler Lösungen zu schließen.

Die Argumentation, wie sie voranstehend benutzt wurde, ist auch im Falle entarteter Eigenwerte $k$ möglich, wobei die Orthogonalitätsbedingungen bezüglich der angepaßten Eigenfunktionen erfüllt werden müssen. Im allgemeinen ergibt sich über die verschiedenen angepaßten Funktionen dann eine Aufspaltung der entarteten Zustände für $\delta k \neq 0$.

Insgesamt ergibt sich also, daß von Null verschiedene diskrete Eigenwerte $k$ des stationären Problems Übergänge zu instabilen Lösungen darstellen, falls nicht alle Lösungen des adjungierten Problems zum selben $k$-Wert orthogonal zur betrachteten Lösung sind.

Liegt ein Kontinuum von Eigenwerten $k$ vor - das ist z. B. möglich, wenn das Gleichgewicht eine Wellenstruktur besitzt (siehe Abschn. 2) -, dann kann es sein, daß die verschiedenen $k$-Werte des Kontinuums verschiedene Zweige der Dispersionsbeziehung darstellen. In diesem Fall gilt der voranstehende Satz auch für kontinuierliche Eigenwerte $k$. Für ein Gleichgewicht mit räumlich periodischer Struktur ist dies in der Tat der Fall. Die Translationsinvarianz ergibt nämlich als Lösungsform für beliebiges $\omega$ für jedes $k$

$$
\Phi_{1}, A_{13}=\exp \left\{i k_{1} q^{1}\right\} \times \text { Period. Funktion von } q^{1}
$$

mit beliebig reellem $k_{1}$. An jede durch $k$ charakterisierte nicht kontinuierlich entartete stationäre Lösung schließt sich also ein Kontinuum nichtstationärer Lösungen an ${ }^{5}$. Ebenso gilt das Umgekehrte, daß bei festgehaltenem nicht kontinuierlich entartetem $k_{1}$ in Abhängigkeit von $k$ ein Kontinuum nichtstationärer Lösungen in der Umgebung einer stationären Lösung existiert. Die im Vorangehenden beschriebene Methode entspricht gerade der Bestimmung dieser Nachbarlösungen.

\section{Beispiele}

\subsection{Das Thermodynamische Gleichgewicht}

Als eine Art Prüfstein für die im Vorangehenden beschriebene Methode werde als erstes das thermodynamische Gleichgewicht betrachtet. Es wird beschrieben durch Verteilungsfunktionen

$$
f_{0}^{(v)} \sim \exp \left\{-\frac{m^{(v)} v^{2}}{2 k T}-\frac{e^{(v)} \Phi_{0}}{k T}\right\} .
$$

Damit ist $\quad j_{0} \equiv 0, \quad \varrho_{0}=e^{(\mathrm{a})} n^{(\mathrm{a})} \exp \left\{-\frac{e^{(\mathrm{a})} \Phi_{0}}{k T}\right\}+e^{(\mathrm{b})} n^{(\mathrm{b})} \exp \left\{-\frac{e^{(\mathrm{b})} \Phi_{0}}{k T}\right\}$,

wobei für $\Phi_{0}=0$ auch $\varrho_{0}$ verschwinden soll. Für $\Phi_{1}$ und $A_{1}$ ergeben sich somit die ungekoppelten Gleichungen

$$
\left(\begin{array}{c}
\partial^{2} \\
\partial y^{2}
\end{array}+\frac{\partial^{2}}{\partial z^{2}}\right) \Phi_{1}=-\left.4 \pi \Phi_{1} \frac{\partial \varrho_{0}}{\partial \Phi_{0}}\right|_{\Phi_{0}=0}=\left(\begin{array}{c}
4 \pi e^{(\mathrm{a}) 2} n^{(\mathrm{a})} \\
k T
\end{array}+\frac{4 \pi e^{(\mathrm{b}) 2} n(\mathrm{~b})}{k T}\right) \Phi_{1}, \quad\left(\begin{array}{c}
\partial^{2} \\
\partial y^{2}
\end{array} \frac{\partial^{2}}{\partial z^{2}}\right) A_{1 z}=0 .
$$

In der Gleichung für $\Phi_{1}$ treten auf der rechten Seite die Debye-Längen $\lambda^{(\mathrm{a})}$ und $\lambda^{(\mathrm{b})}$ der beiden Teilchensorten auf. In $y$-Richtung wird der Ansatz

$$
\Phi_{1} \sim e^{i k y}, \quad A_{1 z} \sim e^{i k y}
$$

gemacht, so daß für $k$ das Eigenwertproblem folgt

$$
\frac{\partial^{2} \Phi_{1}}{\partial z^{2}}=\left(k^{2}+\lambda^{(\mathrm{a})-2}+\lambda^{(\mathrm{b})-2}\right) \Phi_{1}, \quad \frac{\partial^{2} A_{1 z}}{\partial z^{2}}=k^{2} A_{1 z} .
$$

Es existieren also keine von Null verschiedenen positiven Eigenwerte für $k^{2}$, die Instabilität bedeuten würden, was in Übereinstimmung damit ist, daß das thermodynamische Gleichgewicht stabil ist.

${ }^{5}$ Fine kontinuierliche Entartung könnte durch $k_{1}$ beschrie-

ben sein. 


\subsection{Der neutrale ebene $z$-Pinch}

In spezieller Form liegt hierfür eine Diskussion von $\mathrm{F}_{\mathrm{URTH}}{ }^{1}$ vor, der für die eine Teilchensorte die Masse $m=0$ und die Temperatur $T=0$ angenommen hatte. Hier sollen $m^{(a)}$ und $m^{(\mathrm{b})}$ beliebig sein, dagegen wird weiterhin angenommen, daß das Plasma im ungestörten Fall neutral ist. Die ungestörten Verteilungsfunktionen, die eine derartige Situation beschreiben, seien

$$
f_{0}^{(v)} \sim \exp \left\{-\frac{m^{(v)}}{2 k T^{(v)}}\left(v^{2}-2 v^{(v)}\left(v+\frac{e^{(v)}}{m^{(v)} c} A_{0 z}(x)\right)\right)\right\} .
$$

Die Plasmaanordnung ist neutral für $v^{(\mathrm{a})} e^{(\mathrm{a})} / T^{(\mathrm{a})}=v^{(\mathrm{b})} e^{(\mathrm{b})} / T^{(\mathrm{b})}$.

Für die ungestörte Stromdichte ergibt sich somit

demzufolge für $A_{0 z}(x)$ die Gleichung gilt

$$
j_{0 z}=2 e^{(\mathrm{a})} n_{0} v^{(\mathrm{a})} \exp \left\{+\frac{e^{(\mathrm{a})} v^{(\mathrm{a})}}{k T^{(\mathrm{a})} c} A_{0 z}(x)\right\},
$$

$$
\frac{\mathrm{d}^{2} A_{0 z}}{\mathrm{~d} x^{2}}=-2 \frac{4 \pi e^{(\mathrm{a})} n_{0} v^{(\mathrm{a})}}{c} \exp \left\{+\frac{e^{(\mathrm{a})} v^{(\mathrm{a})}}{k T^{(\mathrm{a})}} A_{0 z}(x)\right\} .
$$

Mit

$$
\frac{4 \pi e^{(\mathrm{a}) 2 n_{0}}}{k T^{(\mathrm{a})}} \frac{v^{(\mathrm{a}) 2}}{c^{2}}=\delta^{-2}, \quad x=\delta \xi, \quad \frac{e^{(\mathrm{a})} v^{(\mathrm{a})}}{k T^{(\mathrm{a})} c} A_{0 z}(x)=D(\xi)
$$

geht sie über in

$$
\begin{array}{lc}
\text { geht sie über in } & \mathrm{d}^{2} D / \mathrm{d} \xi^{2}=-2 e^{D} . \\
\text { Die Lösung ist } & D=D_{0}-2 \ln \operatorname{Cos}\left(e^{D_{0} / 2} \xi\right) ;
\end{array}
$$

$D_{0}$ kann dabei ohne Beschränkung der Allgemeinheit Null gesetzt werden.

Ist $D_{1}$ die Störung von $D$ (eine Störung von $\Phi$ muß verschwinden, das ist analog zum vorangehenden Beispiel), so gilt gemäß (7) und (9) die Gleichung

$$
\frac{\partial^{2} D_{1}}{\partial \xi^{2}}+\delta^{2} \frac{\partial^{2} D_{1}}{\partial y^{2}}=-2 D_{1} e^{D}=-2 D_{1} \frac{1}{\operatorname{Cos}^{2} \xi}
$$

$$
\text { und mit } \quad D_{1} \sim e^{i k y}
$$

die hermitesche Eigenwertgleichung $\quad \frac{1}{2} \frac{\partial^{2} D_{1}}{\partial \xi^{2}}+\left(-\frac{1}{2}(k \delta)^{2}+\frac{1}{\operatorname{Cos}^{2} \xi}\right) D_{1}=0$.

Diese Gleichung ist identisch mit FurThs Gleichung ${ }^{1}$ (36).

Man kann sie mit einer Schröprnger-Gleichung vergleichen, wobei

$$
E=-\frac{1}{2}\left(k^{2} \delta^{2}\right) \quad \text { und } \quad U=-1 / \operatorname{Cos}^{2} \xi
$$

Energieeigenwert und potentielle Energie des Teilchens sind, das durch diese Schröbrnger-Gleichung beschrieben wird. Im vorliegenden Fall interessiert die Existenz negativer Eigenwerte für $E$. Da das Minimum von $U$ gleich -1 ist, muß für die Existenz eines Eigenwertes sicher

$$
-E=\frac{1}{2}\left(k^{2} \delta^{2}\right)<1
$$

sein. Der einzige negative Eigenwert für $E$, dem also ein Übergang zu instabilen Lösungen entspricht, ist

$$
E=-\frac{1}{2} \quad \text { mit } \quad D_{1}=1 / \operatorname{Cos} \xi \text {. }
$$

Außerdem ist $E=0$ ein zulässiger Eigenwert, der aber im Rahmen der hier durchgeführten Untersuchungen keine Aussage über Stabilität zuläßt.

„Potentielle Energie“ und Eigenwert sind für einen Vergleich mit späteren Beispielen in Abb. 1 dargestellt. $\delta \rightarrow \infty$ bedeutet Übergang zum thermodynamischen Gleichgewicht, das stabil ist, so daß zu vermuten ist, daß für $k^{2} \delta^{2}<1$ instabiles Verhalten vorliegt.

Abb. 1. Potentielle Energie $U(\xi)=-1 / \operatorname{Cos}^{2} \xi$ und Energieeigenwert $E$ beim neutralen ebenen $z$-Pinch der Gl. (26).

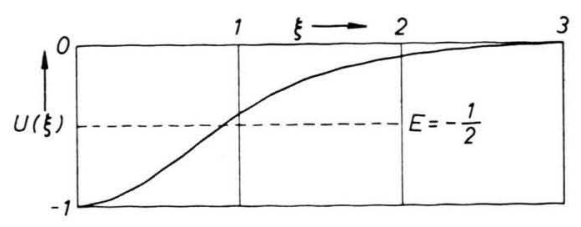




\subsection{Der neutrale ebene $\Theta$-Pinch}

Ein im ungestörten Zustand neutraler $\Theta$-Pinch kann z. B. durch Verteilungsfunktionen folgender Art beschrieben werden

$$
\begin{aligned}
f_{0}^{(v)} & \sim \exp \left[-\frac{m^{(v)}}{2 k T^{(v)}}\left(v^{2}+\gamma^{(v)}\left(v_{2}+\frac{e^{(v)}}{m^{(v)} c} A_{0 z}(x)\right)^{2}\right)-\frac{e^{(v)} \Phi_{0}}{2 k T^{(v)}}\right] \\
& \sim \exp \left[-\frac{m^{(v)}}{2 k T^{(v)}}\left(v_{x}^{2}+v_{y^{2}}+\left(1+\gamma^{(v)}\right)\left(v_{z}+\frac{\gamma^{(v)}}{1+\gamma^{(v)}} \frac{e^{(v)}}{m^{(v)} c} A_{0 z}\right)^{2}\right)-\frac{\gamma^{(v)}}{1+\gamma^{(v)}} \frac{m^{(v)}}{\left.2 k T^{(v)}\left(\frac{e^{(v)}}{m^{(v)} c}\right)^{2} A_{0 z}^{2}-\frac{e^{(v)} \Phi_{0}}{2 k T^{(v)}}\right]}\right.
\end{aligned}
$$

mit

$$
\frac{\gamma^{(\mathrm{a})}}{1+\gamma^{(\mathrm{a})}} \frac{e^{(\mathrm{a}))^{2}}}{m^{(\mathrm{a})} T^{(\mathrm{a})}}=\frac{\gamma^{(\mathrm{b})}}{1+\gamma^{(\mathrm{b})}} \frac{e^{(\mathrm{b}) 2}}{m^{(\mathrm{b})} T^{(\mathrm{b})}}, \quad \Phi_{0}=0 .
$$

Das bedeutet, daß $\varrho_{0}$ identisch in $A_{0 z}$ verschwindet, so daß sich wieder keine Kopplung zwischen $A_{1 z}$ und $\Phi_{1}$ ergibt. Die ungestörte Stromdichte ist

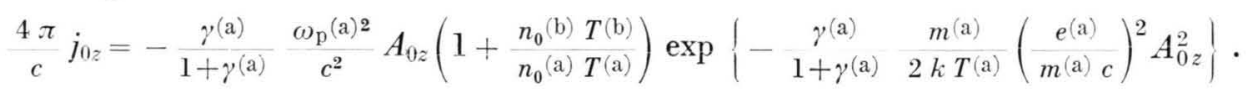

Darin ist $\omega_{\mathrm{p}}{ }^{(\mathrm{a})}$ die Plasmafrequenz der Teilchensorte a bei der Dichte $n_{0}{ }^{(\mathrm{a})}$, die die Dichte bei $x=0$ bedeuten soll; dabei ist $A_{0 z}(0)=0$ angenommen.

Mit

$$
\frac{\gamma^{(\mathrm{a})}}{1+\gamma^{(\mathrm{a})}} \frac{\omega_{\mathrm{p}}^{(\mathrm{a}) 2}}{c^{2}}\left(1+\frac{n_{0}(\mathrm{~b}) T^{(\mathrm{b})}}{n_{0}(\mathrm{a})} T^{(\mathrm{a})}\right)=\delta^{-2}, \quad \frac{x}{\delta}=\xi, \quad \frac{\gamma^{(\mathrm{a})}}{1+\gamma^{(\mathrm{a})}} \frac{m^{(\mathrm{a})}}{2 k T^{(\mathrm{a})}}\left(\frac{e^{(\mathrm{a})}}{m^{(\mathrm{a})} c}\right)^{2} A_{0 z}^{2}=D^{2}(\xi)
$$

ergibt sich für $D(\xi)$ die Gleichung

$$
\frac{\mathrm{d}^{2} D}{\mathrm{~d} \xi^{2}}=D(\xi) \exp \left\{-D^{2}(\xi)\right\}, \quad\left(\frac{\mathrm{d} D}{\mathrm{~d} \xi}\right)^{2}=\left(D_{\infty}^{\prime}\right)^{2}-\exp \left\{-D^{2}\right\}, \quad D_{\infty}^{\prime} \geq 1 ;
$$

$D_{\infty}^{\prime}$ charakterisiert das Magnetfeld für $\xi \rightarrow \pm \infty .1 / D_{\infty}^{\prime 2}$ ist das $\beta$ der Anordnung. Die Lösungen von (42) sind monoton, z. B. monoton wachsend und antimetrisch; asymptotisch gilt $D(\xi) \approx D_{\infty}^{\prime} \xi, \xi \rightarrow \infty$. Für $\beta=\frac{1}{2}$ ist in Abb. 2 die Lösung dargestellt.

Aus (42) ergibt sich für die Störung $D_{1}$ von $D$ bei einem Ansatz $D_{1} \sim e^{i k y}$ für die $y$-Abhängigkeit die hermitesche Eigenwertgleichung

$$
\frac{1}{2}+\frac{\partial^{2} D_{1}}{\partial \xi^{2}}\left(-\frac{1}{2}\left(k^{2} \delta^{2}\right)-\left(\frac{1}{2}-D^{2}(\xi)\right) \exp \left\{-D^{2}(\xi)\right\}\right) D_{1}=0,
$$

so daß wie in Abschn. 2.2 wieder eine „Energie“ $E$ und eine „potentielle Energie“ $U(\xi)$ durch

$$
E=-\frac{1}{2}\left(k^{2} \delta^{2}\right), \quad U(\xi)=\left(\frac{1}{2}-D^{2}(\xi)\right) \exp \left\{-D^{2}(\xi)\right\}
$$

definiert werden können. $U(\xi)$ ist in Abb. 3 qualitativ dargestellt. Der Abszissenmaßstab ist wegen der Eigenschaften von $D$ im wesentlichen $\xi / \sqrt{ } \beta$. Für $\beta \rightarrow 0$ schrumpft die vorhandene Mulde bezüglich $\xi \mathrm{im}$ -

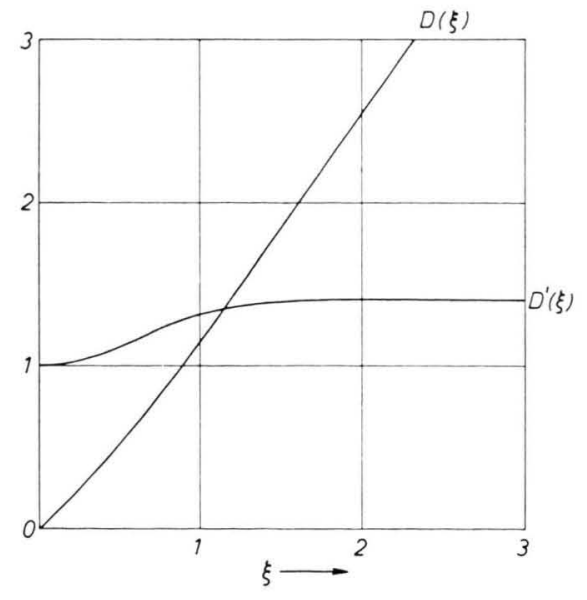

$\leftarrow$ Abb. 2. Vektorpotential $D(\xi)$ und Magnetfeld $D^{\prime}(\xi)$ beim ebenen $\Theta$-Pinch der Gl. (28) für $\beta=\frac{1}{2}$.

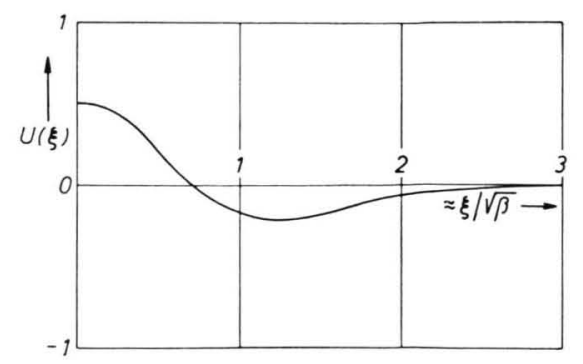

Abb. 3. Potentielle Energie $U(\xi)=\left(\frac{1}{2}-D^{2}(\xi)\right) \exp \left\{-D^{2}(\xi)\right\}$ beim ebenen $\Theta$-Pinch der Gl. (28) (qualitativ). 
mer mehr zusammen, so daß es sicher einen $\beta$-Wert gibt, bei dem es keine negativen Eigenwerte für $E$ bzw. positive Eigenwerte für $k^{2}$ mehr geben kann. Kleines $\beta$ wirkt sich also stabilisierend aus im Sinne der hier durchgeführten Betrachtung. Eine Abschätzung für den niedrigsten Eigenwert für $E$ erhält man nach dem „Korrespondenzprinzip“ (WBK-Methode) durch

$$
\oint \pm \sqrt{2(E-U(\xi))} \mathrm{d} \xi=2 \pi \cdot \frac{1}{2},
$$

wobei über einen Hin- und Hergang der Bahn des „Teilchens“ zu integrieren ist. Wegen des monotonen Verhaltens von $D(\xi)$ kann statt über $\xi$ auch über $D$ integriert werden. Bei Integration nur über einen Hingang ergibt sich also

$$
\int_{D_{0}}^{D_{1}} \frac{\sqrt{E+\left(D^{2}-\frac{1}{2}\right) \exp \left(-D^{2}\right)}}{\sqrt{(1 / \beta)-\exp \left(-D^{2}\right)}} \mathrm{d} D=\frac{\pi}{2 \sqrt{2}},
$$

$D_{0}$ und $D_{1}$ sind untere und obere Nullstelle des Zählers.

Der instabilste Fall ist $\beta=1$, außerdem wird der Maximalwert des Integrals innerhalb des zulässigen $E$-Bereiches für $E=0$ erreicht. Es kann also nur dann Lösungen geben, wenn

$$
\int_{1 / \sqrt{ } 2}^{\infty} \frac{\sqrt{D^{2}-\frac{1}{2}}}{\sqrt{1-\exp \left(-D^{2}\right)}} \exp \left\{-D^{2} / 2\right\} \mathrm{d} D>\frac{\pi}{2 \sqrt{2}}=1,11
$$

ist. - Das Integral läßt sich leicht nach oben abschätzen; mit $D^{2} / 2=x$ geht es über in

$$
\int_{1 / 4}^{\infty} \frac{\sqrt{1-\frac{1}{4} x}}{\sqrt{1-e^{-2 x}}} e^{-x} \mathrm{~d} x<\int_{1 / 4}^{\infty} e^{-x} \mathrm{~d} x=e^{-1 / 4}=0,775 ;
$$

Es ist also etwa um den Faktor 1,43 kleiner als gemäß (47) nötig wäre. Der betrachtete ebene $\Theta$-Pinch ist also nach dieser Abschätzung im Sinne der hier durchgeführten Betrachtung stabil.

\subsection{Der zylindersymmetrische neutrale $\Theta$-Pinch}

Ein neutraler zylindersymmetrischer $\Theta$-Pinch ist nur noch unter sehr speziellen Bedingungen möglich, da man für die Kompensation zweier verschieden vom Ort abhängiger Kräfte, nämlich des thermischen Druckes und der Zentrifugalkraft der Ströme, nicht wie in den vorangegangenen Beispielen mit einem Parameter auskommt, um gleiche Elektronen- und Ionenverteilung zu erhalten. Als ungestörte Verteilungsfunktionen werden gewählt

$$
f_{0}^{(v)} \sim \exp \left[-\frac{m^{(v)}}{2 k T^{(v)}}\left(v_{r}^{2}+v_{z}^{2}+v_{\varphi}^{2}+2 \omega^{(v)} x\left(v_{\varphi}+\frac{e^{(v)}}{m^{(v)} c} A_{0 \varphi}(r)\right)\right)\right] .
$$

Die Ladungsdichte verschwindet identisch in $A_{0 \varphi}$ für

$$
\omega^{(\mathrm{a}) 2} m^{(\mathrm{a})} / T^{(\mathrm{a})}=\omega^{(\mathrm{b}) 2} m^{(\mathrm{b})} / T^{(\mathrm{b})} \quad \text { und } \quad \omega^{(\mathrm{a})} e^{(\mathrm{a})} / T^{(\mathrm{a})}=\omega^{(\mathrm{b})} e^{(\mathrm{b})} / T^{(\mathrm{b})} ;
$$

das ist nur möglich, wenn

$$
T^{(\mathrm{a})} / T^{(\mathrm{b})}=\left(e^{(\mathrm{a}) 2} / e^{(\mathrm{b}) 2}\right)\left(m^{(\mathrm{b})} / m^{(\mathrm{a})}\right)
$$

gilt. Für die ungestörte Stromdichte ergibt sich dann

$$
\left.\frac{4 \pi}{c} j_{0 \varphi}=-\frac{4 \pi}{c} e^{(\mathrm{a})} n_{0} \omega^{(\mathrm{a})} r\left(1+\frac{e^{(\mathrm{b}) 2}}{e^{(\mathrm{a}) 2}} \frac{m^{(\mathrm{a})}}{m^{(\mathrm{b})}}\right) \cdot \exp \left\{-\frac{e^{(\mathrm{a})} \omega^{(\mathrm{a})}}{k T^{(\mathrm{a})} c} r A_{0 \varphi}-\frac{m^{(\mathrm{a})} \omega^{(\mathrm{a}) 2} r^{2}}{2 k T^{(\mathrm{a})}}\right)\right\},
$$

so daß für $A_{0 \varphi}$ die Gleichung besteht

$$
\left.\frac{\mathrm{d}}{\mathrm{d} r} \frac{1}{r} \frac{\mathrm{d}}{\mathrm{d} r} r A_{0 \varphi}=+\frac{4 \pi}{c} e^{(\mathrm{a})} n_{0} \omega^{(\mathrm{a})} r\left(1+\frac{e^{(\mathrm{b}) \mathbf{2}}}{e^{(\mathrm{a}) \mathbf{2}}} \frac{m^{(\mathrm{a})}}{m^{(\mathrm{b})}}\right) \cdot \exp \left\{-\frac{e^{(\mathrm{a})} \omega^{(\mathrm{a})}}{k T^{(\mathrm{a})} c} r A_{0 \varphi}-\frac{m^{(\mathrm{a})} \omega^{(\mathrm{a}) \mathbf{2}} r^{2}}{k T^{(\mathrm{a})}}\right)\right\} .
$$

Die Definitionen

$$
\delta^{4}=\frac{c^{2} k T^{(\mathrm{a})}}{\omega^{(\mathrm{a}) 2} \omega^{2} m^{(\mathrm{a})}}\left(1+\frac{e^{(\mathrm{b}) 2}}{e^{(\mathrm{a}) 2}} \frac{m^{(\mathrm{a})}}{m^{(\mathrm{b})}}\right), \quad \frac{r}{\delta}=\varrho, \quad \frac{e^{(\mathrm{a})} \omega^{(\mathrm{a})}}{k T^{(\mathrm{a})} c} r A_{0 \varphi}(r)=\varrho D(\varrho), \quad \frac{m^{(\mathrm{a})} \omega^{(\mathrm{a})^{2}} \delta^{2}}{2 k T^{(\mathrm{a})}}=\alpha^{2}
$$


führen sie über in

$$
\frac{\mathrm{d}}{\mathrm{d} \varrho} \varrho \frac{\mathrm{d}}{\varrho} \frac{\mathrm{d}}{\mathrm{d} \varrho} \varrho D(\varrho)=+\varrho \exp \left\{-\varrho D+\alpha^{2} \varrho^{2}\right\} .
$$

Entsprechend ergibt sich für die Störung $D_{1}$ von $D$ die Gleichung

was mit dem Ansatz

$$
\begin{aligned}
\frac{\partial}{\partial \varrho} \frac{1}{\varrho} \frac{\partial}{\partial \varrho} \varrho D_{1}+\delta^{2} \frac{\partial^{2} D_{1}}{\partial z^{2}} & =-\varrho^{2} \exp \left\{-\varrho D+\alpha^{2} \varrho^{2}\right\} D_{1}, \\
D_{1} & \sim e^{-i k z}
\end{aligned}
$$

in die hermitesche Eigenwertgleichung

$$
\frac{1}{2}\left(\frac{\partial^{2}}{\partial \varrho^{2}}+\frac{1}{\varrho} \frac{\partial}{\partial \varrho}\right) D_{1}+\left(-\frac{k^{2} \delta^{2}}{2}-\frac{1}{2}\left(-\varrho^{2} \exp \left\{-\varrho D+\alpha^{2} \varrho^{2}\right\}+\frac{1}{\varrho^{2}}\right) D_{1}=0\right.
$$

übergeht. Das entspricht wieder einem quantenmechanischen Problem mit dem Energieeigenwert $E$ und der potentiellen Energie $U$

$$
E=-k^{2} \delta^{2} / 2, \quad U=\left(1 / \varrho^{2}\right)-\varrho^{2} \exp \left\{-\varrho D+\alpha^{2} \varrho^{2}\right\} .
$$

Gl. (55) kann einmal integriert werden: Multiplikation mit $-\left(2 \alpha^{2}-\frac{1}{\varrho} \frac{\mathrm{d}}{\mathrm{d} \varrho}(\varrho D)\right)$ ergibt

$$
\frac{1}{2}\left(\frac{1}{\varrho} \frac{\mathrm{d}}{\mathrm{d} \varrho}(\varrho D)-2 \alpha^{2}\right)^{2}=\frac{1}{2}\left(C-\exp \left\{-\varrho D+\alpha^{2} \varrho^{2}\right\}\right)
$$

oder

$$
2 \frac{\mathrm{d}}{\mathrm{d} \varrho^{2}}\left(\varrho D-\alpha^{2} \varrho^{2}\right)= \pm V C-2 \exp \left\{-\left(\varrho D-\alpha^{2} \varrho^{2}\right)\right\},
$$

woraus

$$
\varrho D-\alpha^{2} \varrho^{2}=-A_{0}-\ln 2+2 \ln \operatorname{Cos}\left(\frac{1}{2} \varrho^{2} \exp \left\{A_{0} / 2\right\}\right), \quad C=4 e^{A_{0}}
$$

folgt, wobei wieder ohne Beschränkung der Allgemeinheit $A_{0}=0$ gesetzt werden darf.

Der damit resultierende Verlauf der potentiellen Energie gemäß Gl. (59) ist in Abb. 4 dargestellt.

Die Gl. (58) für $D_{1}$ geht mit (62) über in $\frac{\mathrm{d}}{\mathrm{d} \varrho}\left(\frac{1}{\varrho} \frac{\mathrm{d}}{\mathrm{d} \varrho} \varrho D_{1}\right)-k^{2} \delta^{2} D_{1}+\frac{2 \varrho^{2}}{\operatorname{Cos}^{2}\left(\varrho^{2} / 2\right)} D_{1}=0$.

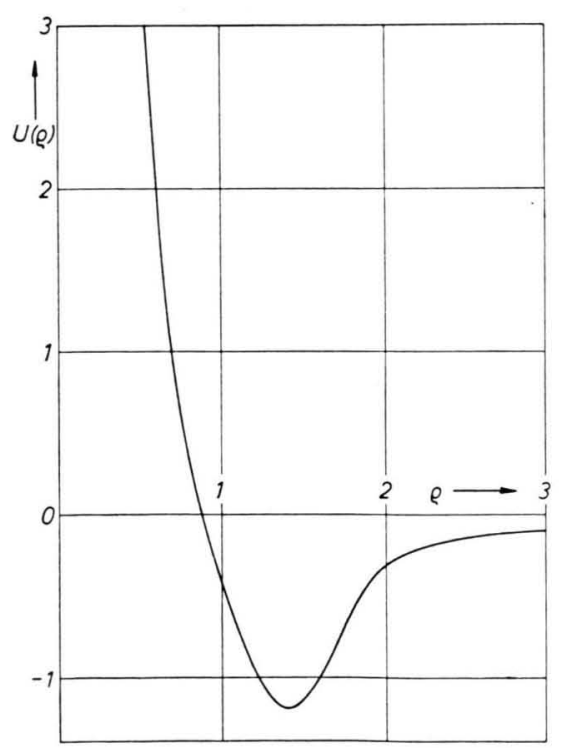

Abb. 4. Potentielle Energie $U(\varrho)=\left(1 / \varrho^{2}\right)-2 \varrho^{2} / \operatorname{Cos}^{2}\left(\varrho^{2} / 2\right)$ beim zylindersymmetrischen neutralen $\Theta$-Pinch der Gl. (49).
Zur Untersuchung dieser Gleichung auf die Existenz positiv reeller Eigenwerte $k$ sei

$$
x=Q^{2} / 2, \quad \varrho D_{1}=y(x)
$$

eingeführt. Aus der Gleichung für $D_{1}$ wird dann

$$
\frac{\mathrm{d}^{2} y}{\mathrm{~d} x^{2}}-\frac{k^{2} \delta^{2} y}{2 x}+\frac{2}{\operatorname{Cos}^{2} x} y=0 .
$$

Für $k^{2} \delta^{2}=0$ sind die beiden unabhängigen Lösungen dieser Gleichung

$$
y_{1}=\operatorname{Tan} x, \quad y_{2}=x \operatorname{Tan} x-1 .
$$

Wegen der Regularitätsforderung bei $x=0$ kann sich eine Lösung für $k^{2} \delta^{2} \neq 0$ nur an $y_{1}$ anschließen, woraus folgt, daß von Null verschiedene positiv reelle Eigenwerte $k^{2} \delta^{2}$ nicht existieren.

Für $k^{2} \delta^{2}=0$ ist die Gleichung für $y$ identisch mit Gl. (34), dort besteht aber keine entsprechende Regularitätsbedingung wie hier, so daß dort der Anschluß an $y_{2}$ möglich ist, der zu dem einen von Null verschiedenen dort zulässigen Eigenwert führt.

$k^{2} \delta^{2}=0$ ist hier gerade noch ein möglicher Eigenwert; mit ihm ist

$$
\begin{aligned}
& D_{1}(\varrho)=\frac{1}{\varrho} \operatorname{Tan} \frac{\varrho^{2}}{\alpha}, \\
& B_{1 z} \sim \frac{\partial D_{1}}{\partial \varrho} \sim-\frac{1}{\varrho^{2}} \operatorname{Tan} \frac{\varrho^{2}}{2}+\underset{\operatorname{Cos}^{2}\left(\varrho^{2} / 2\right)}{1},
\end{aligned}
$$


so daß das Integral

$$
\int_{0}^{\infty} B_{1 z}^{2} \varrho \mathrm{d} \varrho<\infty
$$

ist. Aus $k=0$ kann aber im Rahmen der vorliegenden Untersuchung nicht auf Instabilität geschlossen werden.

\subsection{Der zylindersymmetrische neutrale z-Pinch}

Als ungestörte Verteilungsfunktion wird betrachtet

$$
\begin{aligned}
f_{0}^{(v)} \sim \exp \left[-\frac{m^{(v)}}{2 k T(v)}(\right. & v_{r}^{2}+v_{\varphi}{ }^{2}+v_{z}{ }^{2} \\
& \left.\left.+2 v^{(v)}\left(v_{z}+\frac{e^{(v)}}{m^{(v)} c} A_{0 z}(r)\right)\right)\right] .
\end{aligned}
$$

Wie beim ebenen $z$-Pinch erhält man Neutralität für

$$
v^{(\mathrm{a})} e^{(\mathrm{a})} / T^{(\mathrm{a})}=v^{(\mathrm{b})} e^{(\mathrm{b})} / T^{(\mathrm{b})} .
$$

Die ungestörte Stromdichte ist daher

$\frac{4 \pi}{c} j_{0 z}(r)=2 \frac{4 \pi e^{(\mathrm{a})} n_{0} v^{(\mathrm{a})}}{c} \cdot \exp \left\{\frac{e^{(\mathrm{a})} v^{(\mathrm{a})}}{k T^{(\mathrm{a})} c} A_{0 z}(r)\right\}$,

so daß für $A_{0 z}$ die Gleichung gilt

$\frac{1}{r} \frac{\partial}{\partial r} r \frac{\partial}{\partial r} A_{0 z}=-2 \frac{4 \pi e^{(\mathrm{a})} n_{0} \frac{v^{(\mathrm{a})}}{c}}{c}$

die mit

$$
\cdot \exp \left\{\frac{e^{(\mathrm{a})} v^{(\mathrm{a})}}{k T^{(\mathrm{a})} c} A_{0 z}(r)\right\},
$$

$$
\begin{aligned}
& \frac{4 \pi \boldsymbol{e}^{(\mathrm{a}) 2} n_{0}}{k T^{(\mathrm{a})}} \frac{v^{(\mathrm{a}) 2}}{c^{2}}=\delta^{2}, \quad r=\delta \varrho, \\
& \frac{e^{(\mathrm{a})} v^{(\mathrm{a})}}{k T^{(\mathrm{a})} c} A_{0 z}(r)=D(\varrho)
\end{aligned}
$$

$$
\text { in } \quad \frac{1}{\varrho} \frac{\partial}{\partial \varrho} \varrho \frac{\partial}{\partial \varrho} D=-2 e^{D}
$$

übergeht. Mit $\varrho=e^{x} \quad$ (75) erhält man daraus

$$
\frac{\partial^{2}}{\partial x^{2}} D=-2 e^{D+2 x}, \quad \frac{1}{2}\left(\frac{\partial D}{\partial x}+2\right)^{2}=C-2 e^{D+2 x}
$$

und damit

$$
D=-2 x+D_{0}-2 \ln \operatorname{Cos}\left(e^{D_{0} / 2} x\right),
$$

wobei $D_{0}=0$ gesetzt werden kann. Für $e^{D}$ ergibt sich also der Ausdruck

$$
e^{D}=\frac{1}{\varrho^{2}} \frac{1}{\operatorname{Cos}^{2} \ln \varrho} .
$$

Für die Störung $D_{1}$ von $D$ gilt somit die Gleichung

$$
\left(\frac{1}{\varrho} \frac{\partial}{\partial \varrho} \varrho \frac{\partial}{\partial \varrho}+\frac{1}{\varrho^{2}} \frac{\partial^{2}}{\partial \varrho^{2}}\right) D_{1}=-2 e^{D} D_{1} \text {, }
$$

was mit dem Ansatz $D_{1} \sim e^{i m \varphi}$

in die hermitesche Eigenwertgleichung

$$
\left(\frac{1}{\varrho} \frac{\partial}{\partial \varrho} \varrho \frac{\partial}{\partial \varrho}-\frac{m^{2}}{\varrho^{2}}\right) D_{1}=-2 e^{D} D_{1}
$$

übergeht. Mit $\varrho=e^{x} \quad$ (82) erhält man daraus

$$
\left(\frac{\partial^{2}}{\partial x^{2}}-m^{2}\right) D_{1}=-2 e^{D+2 x} D_{1}=\frac{-2}{\operatorname{Cos}^{2} x} D_{1} .
$$

Diese Gleichung ist identisch mit (34), so daß gemäß (37) genau 2 erlaubte Eigenwerte $m^{2}$ existieren

$$
m^{2}=0, \quad m^{2}=1 .
$$

Wegen der Diskretheit von $m$ kann die Betrachtung unter 1.2 hier nicht angewendet werden. Es kann also aus $m^{2}=1$ nicht auf Instabilität geschlossen werden. Durch einen Grenzübergang zum thermodynamischen Gleichgewicht wird auch eher der umgekehrte Schluß nahegelegt. Dieser Grenzübergang kann durchgeführt werden, wenn man entsprechend der Bedeutung von $m$

$$
m \sim 2 \pi \delta / \lambda
$$

setzt, wobei $\lambda$ die Wellenlänge am „Plasmarand“ $\delta$ ist. $\delta \rightarrow \infty$, also auch $m \rightarrow \infty$, ist daher stabil. Durch die vorliegende Rechnung ist also keine Instabilität angezeigt.

\subsection{Einige allgemeine Aussagen für ebene Geometrien ohne Raumladung}

Im ebenen Fall folgt nach (8) $\varrho_{0}$ und $j_{0}$ aus einer erzeugenden Funktion, die nur von $\Phi_{0}$ und $A_{03}$ abhängt und nicht mehr explizit von der Koordinate $q^{1}$. Wenn dann identisch in $A_{03}$ gilt

$$
\varrho_{0}=-\left.\frac{\partial W\left(\Phi_{0}, A_{03}\right)}{\partial \Phi_{0}}\right|_{\Phi_{0}=0} \equiv 0,
$$

dann gibt es keine Kopplung zwischen $A_{13}$ und $\Phi_{1}$. $A_{03}$ befolgt eine "klassische Bewegungsgleichung“ mit der "potentiellen Energie“

$$
W\left(A_{03}\right)=-(4 \pi / c) W\left(0, A_{03}\right)
$$

der Form

$A_{03}^{\prime \prime}\left(q^{1}\right)=-\frac{\partial W\left(A_{03}\right)}{\partial A_{03}}, \frac{1}{2} A_{03}^{\prime 2}+W\left(A_{03}\right)=W_{0} ;$

dabei entspricht $q^{1}$ der Zeit. Die Gleichung für $A_{13}$

$$
\frac{1}{2} A_{1}^{\prime \prime}+\left(-\frac{k^{2}}{2}+\frac{1}{2} \frac{\partial^{2} W\left(A_{03}\right)}{\partial A_{03}^{2}}\right) A_{1}=0
$$

ist hermitesch und entspricht einer ScHRöDINGERGleichung mit

$$
E=-\frac{1}{2} k^{2}, \quad U=-\frac{1}{2}\left(\partial^{2} W / \partial A_{0}{ }^{2}\right) .
$$

Damit negative Eigenwerte $E$ existieren können, muß wenigstens in einem gewissen Bereich $\partial^{2} W / \partial A_{0}{ }^{2}$ positiv sein. Es gibt nun Magnetfeld-Plasmakonfigurationen, bei denen $\partial^{2} W / \partial A_{0}{ }^{2}$ immer größer als 
Null ist und auch im Limes großer $x$ nicht nach Null strebt, das sind gewisse räumlich periodische Konfigurationen (siehe Abb. 5). In diesem Fall gibt es im allgemeinen für $E$ Eigenwertskontinua.

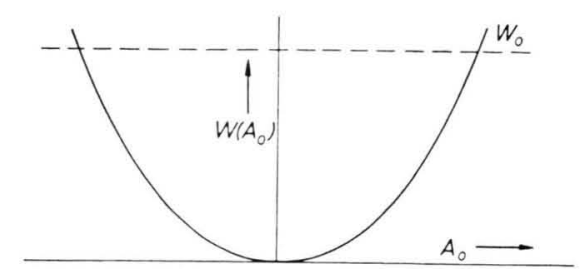

Abb. 5. Die erzeugende Funktion $W\left(A_{0}\right)$ für räumlich periodische Strukturen.

Liegen sie wenigstens zum Teil bei negativen Energien, dann sind sie nach den Ausführungen in Abschn. 1.2 instabil; das gilt also insbesondere für rein sinusförmige Strukturen, die durch $W \sim A_{0}{ }^{2}$ beschrieben werden.

Auch beim $z$-Pinch kann die zweite Ableitung von $W$ stets positiv sein, sie muß aber für $\left|q^{1}\right| \rightarrow \infty$ gegen Null gehen (siehe Abb.6), so daß nicht not-

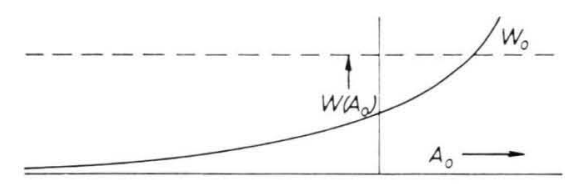

Abb. 6. Die erzeugende Funktion $W\left(A_{0}\right)$ für ebene $z$-Pinche.

wendig negative „Energieeigenwerte“ existieren; wenn sie existieren, dann sind sie jedenfalls diskret. Beim ebenen $\Theta$-Pinch kann schließlich $\partial^{2} W / \partial A_{0}{ }^{2}$ nicht mehr überall positiv sein (siehe Abb. 7). Da

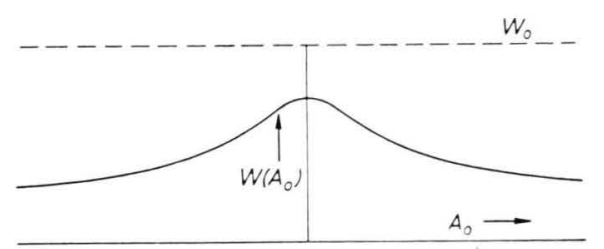

Abb. 7. Die erzeugende Funktion $W\left(A_{0}\right)$ für ebene $\Theta$-Pinche.

das „klassische" Teilchen, dessen Geschwindigkeit das ungestörte Magnetfeld bestimmt, sich sehr langsam im Bereich negativen $\partial^{2} W / \partial A_{0}{ }^{2}$ bewegt, gibt es für das quantentheoretische Teilchen gegebenenfalls einen dicken Potentialberg, so daß bei Vorliegen eines negativen Eigenwertes dieser fast 2-fach entartet sein wird.

Für den ebenen $\Theta$-Pinch gilt auch ganz allgemein, daß sich kleines $\beta$ stabilisierend im Sinne der vor- liegenden Theorie auswirkt. Für sehr kleines $\beta$ folgt nämlich aus (88)

$$
A_{03}^{\prime} \approx \sqrt{2 W_{0}}=B_{2}(\infty), \quad A_{03} \approx B_{2}(\infty) q^{1},
$$

und etwas genauer durch Iteration der Gl. (88)

$$
B_{z}{ }^{2}\left(q^{1}\right)=B_{z}{ }^{2}(\infty)-2 W\left(B_{z}(\infty) q^{1}\right) .
$$

Führt man

$2 W\left(A_{03}\right)=\beta B_{z}{ }^{2}(\infty) f\left(A_{03} / B_{z}(\infty)\right), \quad f \geqq 0$,

an Stelle von $W$ ein und ist $f(0)=1$ der Maximalwert von $f$, dann ist

$$
p\left(q^{1}\right)=\beta\left(B_{z}^{2}(\infty) / 8 \pi\right) f\left(q^{1}\right)
$$

die Druckverteilung und $\beta$ ist das normale Plasma- $\beta$, definiert durch

$$
\beta=\frac{p_{\max }}{B_{2}{ }^{2}(\infty) / 8 \pi}
$$

Weiter folgt aus (93)

$$
\partial^{2} W / \partial A_{03}^{2}=\beta\left(\partial^{2} f\left(q^{1}\right) /\left(\partial q^{1}\right)^{2}\right),
$$

so daß eine „Potentialmulde“ für das „SchröningerTeilchen " für $\beta \rightarrow 0$ beliebig klein wird. Für $\beta \rightarrow 0$ kann es also beim ebenen $\Theta$-Pinch keine stationären Nachbarlösungen geben, die einen Übergang zu instabilen Lösungen darstellen.

\subsection{Einige allgemeine Aussagen für ebene Geometrien ohne Ströme}

Wenn $j_{03}$ identisch in $A_{03}$ verschwindet und $\varrho_{0}$ unabhängig von $A_{03}$ ist, dann reduzieren sich die Gln. (9) zusammen mit (10) auf eine Gleichung für $\Phi_{1}$, die im ebenen Fall unter Berücksichtigung von (8) folgendes Aussehen hat:

$$
\frac{1}{2} \frac{\mathrm{d}^{2} \Phi_{1}}{\left(\mathrm{~d} q^{1}\right)^{2}}+\left(E+\frac{1}{2} \frac{\mathrm{d}^{2} W\left(\Phi_{0}\right)}{\mathrm{d} \Phi_{0}{ }^{2}}\right) \Phi_{1}=0
$$

mit $4 \pi W\left(\Phi_{0}, A_{03}\right)=-W\left(\Phi_{0}\right), E=-k^{2} / 2$. (98) $\Phi_{0}$ befolgt eine „klassische Bewegungsgleichung“

$$
\mathrm{d}^{2} \Phi_{0} /\left(\mathrm{d} q^{1}\right)^{2}=-\mathrm{d} W\left(\Phi_{0}\right) / \mathrm{d} \Phi_{0} .
$$

Damit besteht volle Analogie zu den in 2.6 diskutierten Fällen, so daß hier die entsprechenden Aussagen wie dort gelten. Insbesondere ist also auch eine große Klasse stationärer elektrostatischer Wellen, wie sie von Bernstein, Green und Kruskal ${ }^{1}$ angegeben wurden, instabil. Dabei ist aber zu beachten, daß diese Instabilität sich auf 2-dimensionale Störungen bezieht, bei denen im zeitabhängigen Fall auch Magnetfelder auftreten können, und nicht auf eindimensionale, rein elektrostatische Störungen.

Herrn Prof. Dr. A. Schlüter danke ich herzlich für wertvolle Diskussionen. 